\title{
Optimized formation control of multi-agent system using PSO algorithm
}

\author{
Ahmed M. Hasan, Safanah M. Rafaat \\ Department of Control and Systems Engineering (CSE), University of Technology (UOT), Iraq
}

\begin{tabular}{l} 
Article Info \\
\hline Article history: \\
Received Jan 2, 2020 \\
Revised May 4, 2020 \\
Accepted Jun 11, 2020 \\
\hline
\end{tabular}

\section{Keywords:}

Consensus algorithm

Formation control

Graph theory

Multi-agent system

Particle swarm optimization

\begin{abstract}
Formation Control (FC) is an important application for Multi-agent Systems (MASs) in coordinated control and especially for Unmanned Aerial Vehicle (UAV) which are widely used nowadays in military and civil sections. FC is mostly applied in conjunction with consensus algorithm. In this paper, a framework for an implementation of consensus FC that involves the decentralized type of network control is considered in order to achieve formation keeping, where the control of each vehicle is calculated dependent upon locally existed facts. The dynamic behavior of each vehicle agent is governed by its second-order dynamic model, and the networked mobile vehicle system is modeled by a directed graph. Then, Particle Swarm Optimization (PSO) is implemented for speeding up the convergence to the desired geometrical shape. Acceleration of the network while approaching the coveted shape is achieved and omissions of undesired swing that transpires through the acceleration is examined. The merits and effectiveness of the applied approach are demonstrated using two different examples.
\end{abstract}

Copyright $(0) 2020$ Institute of Advanced Engineering and Science. All rights reserved.

\section{Corresponding Author:}

Ahmed Mudheher Hasan,

Department of Control and Systems Eng.,

Sinaa Street, University of Technology, Iraq.

Email:60163@uotechnology.edu.iq

\section{INTRODUCTION}

Formation control of Multi-agent System (MAS) for multiple Unmanned Aerial Vehicle (UAV) systems has been a hot research area in last decades due to its wide potential applications in various practical fields, such as the incorporation of multiple UAV navigation for infrastructure inspection tasks [1], source seeking [2], localization [3], wheeled mobile robot [4], mini-satellite [5], underwater vehicles and drones as well [6]. In the literature, a considerable problems related to formation control of UAV are discussed in [7]. Actually, formation control is considered as one of the vital issues in cooperative control of multi-UAV systems since it requires keeping coherence between these vehicles from position respective to keep a certain geometric configuration. Although some conventional formation control algorithms that implicate leader-follower, virtual structure, and behavior-based which can be applied with formation control dilemma of multiple UAV systems [8,9], designing a formation control to deal with this issues is still a very interested topic. The previous work implicates several complexities; the existent algorithms cannot adopt when altering the network topology through adding or removing some agents. Another important issue is the speed of constructing the desired formation.

In this sense, a related work about MAS utilizing Sliding Mode Control (SMC) has been reported in [10]. Moreover, a nonlinear Model Prediction Control (MPC) has been also proposed in [11] for formation control. On one hand, Proportional Integral Derivative (PID) algorithm had been proposed by Ling et al., [12] in order to boost the convergence speed of the CA through implementing an incremental PID algorithm, which takes the advantage of full use of the adjacent state of the moving vehicles to substitute 
the proportional feedback gain to improve the speed. However, PID values are selected by trial and error which makes it impractical for real time implementation. On the other hand, Chun et al.; [13] main objective is to enhance the consensus algorithm (CA) and boost the convergence speed of the MAS. Unfortunately, the parameters of PID algorithm had been chosen using Genetic Algorithm (GA) which require a long time for reaching the optimum values. Moreover, a control protocol has been designed by Jinfeng et al.; [14]. The protocol intends to accretion the convergence speed of consensus.

Advance development of consensus theory [15-17], lead the researchers to a fact that consensus delineation can be used to examine the existence of formation control problems in MAS. Ren [18] focus on formation control issues for second-order MAS utilizing consensus based protocols which proves that leader follower and behavior based formation organizations can be considered as a special case of consensus strategy.

A Super Twisting (ST) type controller is utilized for implementing a distributed formation flight for MAS in [19]. Two specified controllers are conducted for navigation purpose named by a PID and ST controllers in order to reach to the correct formation state. A consensus algorithm is chosen to perform the formation flight while the suggested controllers are verified by conducting real-time experiments. However, the consensus was very slow which make it very critical for real time implementation in some cases.

Liu et al., [6] suggest a distributed formation control for obtaining the formation state through considering four multi-rotors in two-and-three dimensional environments. The suggested controller use a fast model predictive control method for various formation cases such as triangular, rectangular, and circular shapes. On the other hand, in [5], Xiwang et al.; study the effect of formation containment control situation for various multi-rotors UAV. The results shows that the states of the UAV followers keep certain formation specified by the convex combination instead of just converge to the convex formed by the UAV leaders. Formation process is verified experimentally through using five quad-rotor UAV's.

In this work, the Consensus Algorithm (CA) has been accelerated using a powerful optimization method called Particle Swarm Optimization (PSO) that can be reviewed in [20-22] and then used to configure the Formation control with Graph Theory (GT) for Multi-Agent System. The rest of the paper is organized as follows: the basic concepts on Graph Theory (GT) and a definition on the problem formulation is presented in section 2, the stability of the networked MAS is discussed in section 3, while the simulation results and the improvement of the optimized performance is presented in section 4 and 5, respectively. Finally, the conclusions about the obtained results are summarized at the end of this paper.

\section{PRELIMINARIES AND PROBLEM FORMULATION}

Commonly, an agent points to a dynamic system. In this paper, the idiom 'agents' is interchangeable with 'Unmanned Aerial Vehicle', which coincide to a nonlinear dynamic system. The basic concepts in Graph Theory (GT) are presented in this section with the problem formulation as well.

\subsection{Basic Concepts in Graph Theory}

A directed graph $G$ can be designated by $G_{d}\left\{V_{e}, E_{d}\right\}$ where each vertices and edges can be represented as a vector $V_{e}=(1, \ldots, N)$ while $E_{d} \subseteq V_{e} \times V_{e}$, respectively. An initial condition to apply consensus algorithm is that digraph must be as "directed spanning tree" model. Consequently, a two substantial roles that must be satisfied in order to establish a communication among agents in MAS [23]:

Rule 1 (connectivity): which means that the directed spanning tree model must be strongly connected.

Rule 2 (Balancing): where the directed spanning tree must be accurately balanced.

\section{Definition 1:}

The communication among any node in the network with its close neighbors is represented by the adjacency matrix called $A$ for each specific directed graph $G_{d}$. On the other hand, the adjacency matrix of a directed graphs $G_{d}$ with an $n$ nodes of an $n \times n$ matrix. Since the $i$, and $j$ elements are equal to one if nodes $i$ and $j$ are neighbors and zero otherwise [24]. The elements of adjacency matrix of $G_{d}$ can be expressed as:

$$
a_{i j}=\left\{\begin{array}{llr}
1 & \text { if } & e_{i j} \in E \\
0 & \text { if } & \text { else }
\end{array}\right.
$$

$a_{i j}:$ is the input for adjacency matrix.

$e_{i j}:$ is the edge through node $i$ to $j$. 


\section{Definition 2:}

Laplacian matrix could be easily computed through the weighted graph as in (2).

$$
l_{i i}=\sum_{j=1, j \neq i}^{n} a_{i j}
$$

\subsection{Problem Formulation}

Formation control is the procedure of designing a specific controller for each vehicle, depending on its individual state and its related state information among its neighbors, which leads the intended group of vehicles into formation state. As an example, consider a group of $M$ vehicles in the specified network and they share an identical dynamic system as represented in (3) [25].

$$
\dot{x_{i}}=A_{v e c} x_{i}^{\mathrm{var}}+B_{v e c} u_{i}^{i n}, \quad i=1, \ldots \ldots, M ; \quad x_{i} \in \mathfrak{R}^{2 n}
$$

where $x_{i}^{\mathrm{var}}$ represents $n \mathrm{c}$ configuration variables for $i$ vehicles, $u_{i}^{i n}$, exemplify the control inputs. For simplicity, we assume the matrices $A_{v e c}$ and $B_{v e c}$ have the following forms

$$
\begin{aligned}
& A_{v e c}=\operatorname{diag}\left(\left(\begin{array}{cc}
0 & 1 \\
a_{21}^{1} & a_{22}^{1}
\end{array}\right), \ldots \ldots \ldots . .\left(\begin{array}{cc}
0 & 1 \\
a_{21}^{n c} & a_{22}^{n c}
\end{array}\right)\right) \\
& B_{v e c}=I_{n c} \otimes\left(\begin{array}{l}
0 \\
1
\end{array}\right)
\end{aligned}
$$

where $I_{n c}$ represent the configuration identity matrix while $\otimes$ represents the Kronecker product.

\section{Definition 3:}

$$
\text { A formation could be designated as a vector } h_{f}=h_{v} \otimes\left(\begin{array}{l}
0 \\
1
\end{array}\right) \in \mathfrak{R}^{2 n M} \text { where } v \text { represent an } M
$$

vehicles in the formation state $h_{f}$ at instant time $t$ [25]. Two factors should be taken into consideration in order to reach convergence of vehicles which are the difference institutions of position and the formation vector. Assume that $r$ and $s$ be two vectors, where $r$, and $s \in \mathfrak{R}^{n}$ can be represented such that:

$$
\begin{aligned}
& x_{p i}(t)-h_{p i}=r \\
& x_{v i}(t)=s
\end{aligned}
$$

The convergence state happen whenever $r(\cdot)$, and $s(\cdot)$ exist such that $x_{p i}(t)-h_{p i}-r(t)=0$ and $x_{v i}(t)-s=0$ as $t$ approach to $\infty$ for $i=1, \ldots ., M$; the concept of the interpretation between vectors in the definition can be explained in Figure 1.

Based on the directed GT discussed in [23], if it is assumed that vehicle $j$ is a neighbor to a different vehicle $i$ and hence the later vehicle $i$ is transmitting information of the first vehicle $j$ by means of a communication link, consequently, the vehicle $j$ is able to get advantages of this information as a feed-back formula to maintain its current state. The neighbors of vehicle $i$ symbolized by $\rrbracket i$ [26]. In this paper, the decentralized stain of the feed-back control law has been implemented, since every vehicle knows only prorated information about its neighbor's $\rrbracket i$. Then assume $u_{i}$ be the control whom its functions of $x_{j}-x_{i}$ with $h_{j}-h_{i}$ for each $j \in \mathbb{D} i$. 


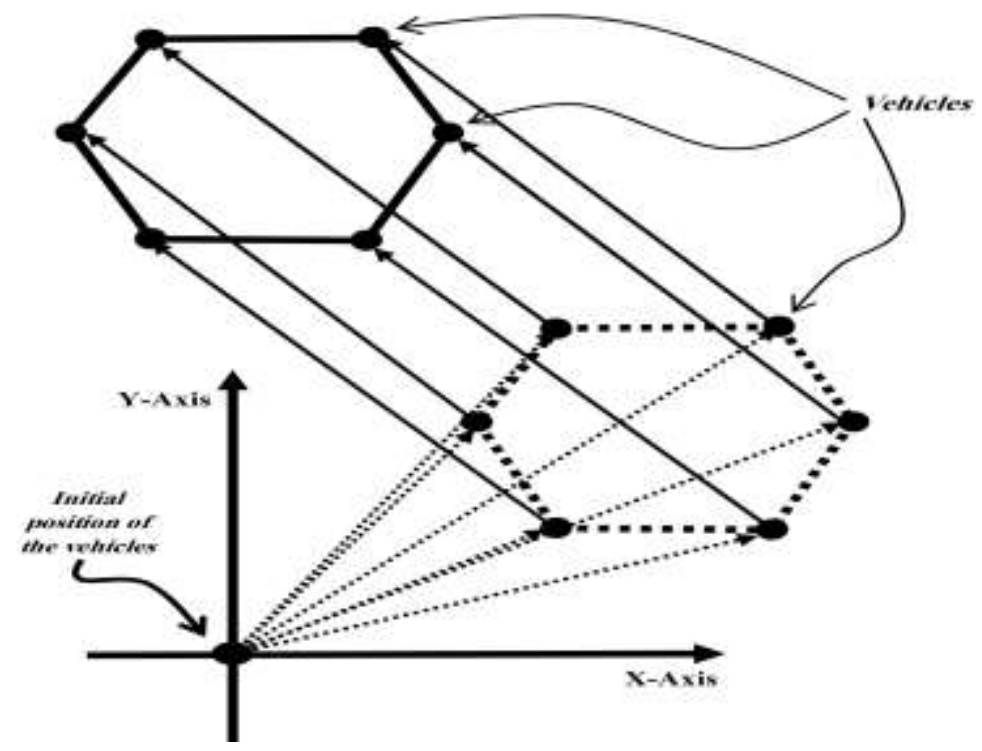

Figure 1. Vehicles movement in the formation state [25]

Thus, the output functions $y_{i}$ are calculated as the average of the comparative displacements and

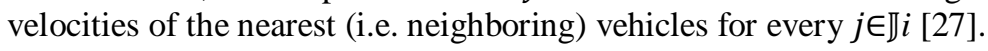

$$
y_{i}=\left(x_{i}^{\text {var }}-h_{i}\right)-\frac{1}{\left|\mathbb{J i}_{i}\right|} \sum_{j \in \mathbb{N}_{i}}\left(x_{j}^{\text {var }}-h_{j}\right), \quad i=1, \ldots, N
$$

Reaching to formation state can be guaranteed through adjusting the state of the feed-back formula accurately. Moreover, the feed-back rule will be used for all vehicles within a specific decentralized control function [28]:

$$
\begin{aligned}
& \boldsymbol{F}_{\boldsymbol{F B}}=I_{M} \otimes \boldsymbol{F}_{\text {vec }} \\
& \boldsymbol{F}_{\text {vec }}=I_{M} \otimes\left(f_{1}, f_{2}\right)
\end{aligned}
$$

where $f_{1}$, and $f_{2}$ are the feed-back coefficients (i.e. gains) that are necessary to ensure convergence to a formation state, $\boldsymbol{F}_{\boldsymbol{F} \boldsymbol{B}}$ is a feed-back matrix, and $I_{M}$ is Indented vector.

$$
\dot{x}=A x+B F_{F B} L(x-h)
$$

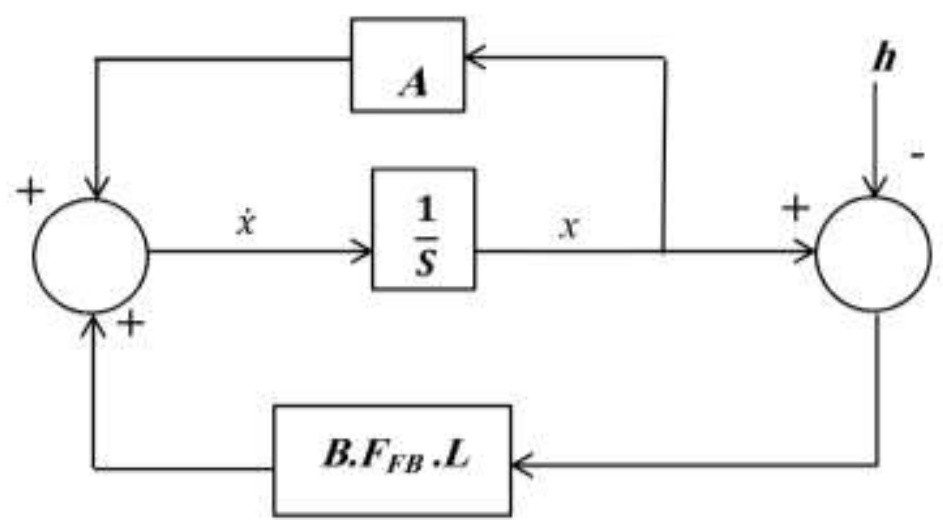

Figure 2. A block diagram for a decentralized feed-back formation control system 


\section{STABILITY CONDITIONS FOR THE NETWORKED MAS}

Suppose that every vehicle in the Multi-agent System (MAS) has a form $A_{v e c}$ and $B_{v e c}$, as illustrated in (4) and (5). In order to investigate the stability of the feed-back matrices. Then, a prior calculation of the eigenvalue of $A_{v e c}+B_{v e c} F_{v e c}$ should be examined through utilizing the Routh's stability conditions. Meanwhile, the examined conditions will be used for the feed-back coefficient's (i.e. gains) $f_{l}$, and $f_{2}$ of $F_{\text {vec }}=I_{M} \otimes\left(f_{1}, f_{2}\right)$ in order to reach to the formation state for each vehicle.

Taking into account the polynomial $r(x)=x^{2}+w x+p$, where $w=w_{1}+w_{2}^{i}$ with $p=p_{1}+p_{2}^{i}$, where as $\mu$ is a root of $r(x), \bar{\mu}$ is a root of $q(x)=x^{2}+w x+p$. Thence, $r(x)$ is considered a stable case if and only if $h_{f}(x)=r(x) . q(x)$. Therefore, the substantial and adequate conditions for stability of $r(x)$ after applying Routh's stability criterion to $h_{f}(x)$ is listed as [29]:

$$
\begin{aligned}
& w_{1}>0 \\
& 2 p_{1}+w_{1}^{2}+w_{2}^{2}>0 \\
& w_{1} p_{1}+w_{2} p_{2}>0 \\
& p_{1} w_{1}^{2}+p_{2} w_{1} w_{2}-p_{2}^{2}>0
\end{aligned}
$$

The earlier conditions will guarantee that the feed-back coefficients $\left(f_{1}, f_{2}\right)$ have a real scalar values, and the polynomials of $A+\lambda B F_{F B}$ have roots with negative real part. Let $u=F_{F B} L\left(x-h_{f}\right)$, where $F_{F B}=I_{n c M} \otimes\left(f_{1}, f_{2}\right)$ while $L=L_{G} \otimes I_{n c}$. Finding both $f_{1}$ and $f_{2}$ solves the stability problems such that $A+\lambda B F_{F B}$ is Hurwitz for each non-zero eigenvalue $\lambda$ of $L_{G}$. After assuming that every coordinates has exact same dynamic Equations [25], this result to the following:

$$
\begin{aligned}
& A+\lambda B F_{F B}=I_{n c M} \otimes\left(\left(\begin{array}{cc}
0 & 1 \\
0 & a_{22}
\end{array}\right)+\lambda\left(\begin{array}{l}
0 \\
1
\end{array}\right)\left(f_{1}, f_{2}\right)\right) \\
& A+\lambda B F_{F B}=I_{n c M} \otimes\left(\begin{array}{cc}
0 & 1 \\
\lambda f_{1} & a_{22}+\lambda f_{2}
\end{array}\right)
\end{aligned}
$$

Meanwhile, the eigenvalues of $A+\lambda B F_{F B}$ are the roots of the given polynomial $r(x)=x_{2}+\left(-a_{22}-\lambda f_{2}\right) x+\left(-\lambda f_{1}\right)$. Moreover, through adopting $\lambda=\delta+B_{i}$ and substituting it in the previous equation will result in a stable polynomial [30].

$$
\begin{aligned}
& -a_{22}-\delta f_{2}>0 \\
& -2 \delta f_{1}+\left(a_{22}+\delta f_{2}\right)^{2}+\omega^{2} f_{2}^{2}>0 \\
& a_{22} \delta f_{1}+\left(2 \delta+\omega^{2}\right) f_{1} f_{2}>0 \\
& -\delta f_{1}\left(a_{22}+\delta f_{2}\right)^{2}-\omega^{2} f_{1} f_{2}\left(a_{22}+\delta f_{2}\right)-\omega^{2} f_{1}^{2}>0
\end{aligned}
$$

Through choosing values for $f_{1}<0$ and $f_{2}<0$ then the conditions are satisfied and it can be noticed that $f_{2}$ is adequately larger in absolute value since $\delta>0$. Accordingly, it can be notice that choosing these values results surly in a stable formation state. This can be more obvious in case of assuming $a_{22}=0$, which leads that each coordinate can be modeled as a double integrator. This results in very concise and simple conditions as:

$$
f_{1} \text { and } f_{2}<0
$$




$$
\frac{f_{2}^{2}}{f_{1}}<-\frac{\omega^{2}}{\delta\left(\delta^{2}+\omega^{2}\right)}
$$

This condition can be accomplished for small values of $f_{l}$ and $f_{2}$.

\section{SIMULATION RESULTS}

The goal of this paper is to implement the consensus formation control that includes the decentralized Multi-agent System (MAS) and to improve the performance of reaching the desired formation pattern through conducting two main shapes. First shape of formation is circle; while the second shape of formation is hexagon. Assume $a_{22}=0$ in (16) in order to treat the coordinate as double integrator. The Laplacian matrix in this paper is dynamically generated for any two adjacent vehicles in the formatted model satisfying the condition that state as the distance between two adjacent vehicles is less than a specific value. According to the previous conditions, $f_{l}$ and $f_{2}$ are randomly selected in order to satisfy the substantial conditions for stability. On the other hand, the rapport for the two communication topologies is represented:

$$
x=I_{N} \otimes A_{v e c} x+L_{G} \otimes B_{v e c} F_{v e c}(x-h)
$$

The two cases supposed in this paper will be discussed later.

First Case: Circle formation

Twelve vehicles are involved in the first case in order to perform the circle formation in this case. The initial positions for the generated vehicles are chosen randomly at first time in the specified region as shown in Figure 3. Through implementing the formation control which are represented by (24) then the vehicles will move accordingly until reach its final position (i.e. desired) in the formatted circle, as shown in Figure 4. The step by step movement for the twelve vehicles starting from the initial states for positions to the final positions are shown in Figure 5. It is very clear that the vehicles converge to the desired formation. In this figure, the initial position states of the vehicles are marked with an 'o' while the final positions of the circle formation are marked with '*'.

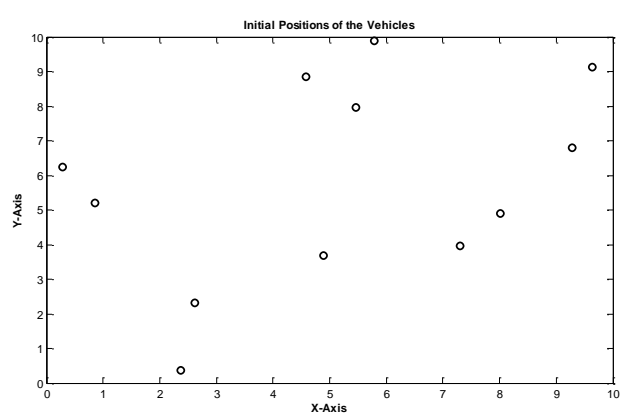

Figure 3. Twelve vehicles at random initial positions

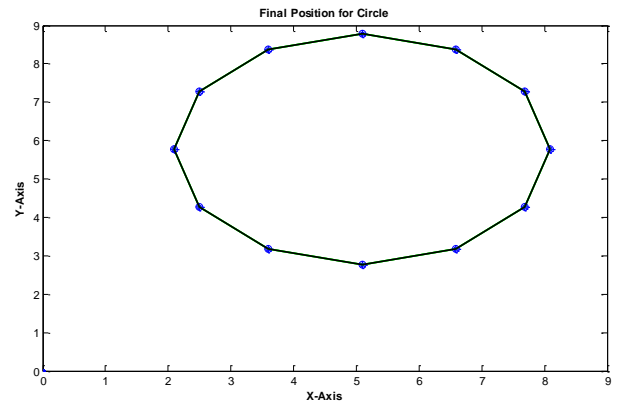

Figure 4. Twelve vehicles in the final formation positions

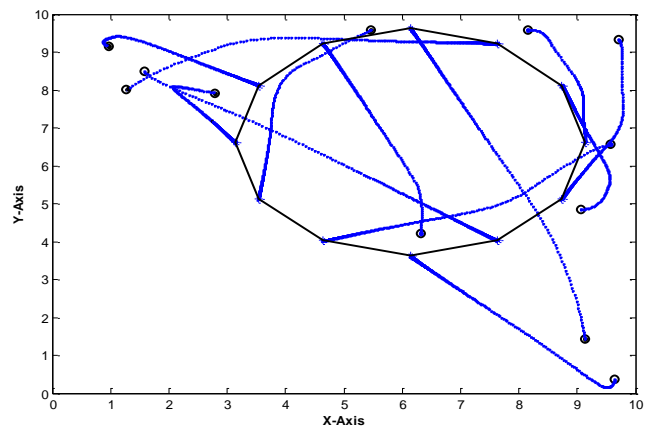

Figure 5. Trajectory of the twelve vehicles in the circle formation 
Second Case: Hexagon formation

In order to validate the formation control strategies for the Multi-agent System (MAS). Then, another formation case is considered which represent a hexagon formation which consist of six vehicles. Same initial conditions are considered as in previous formation example where the initial positions of the vehicles are randomly chosen at a specific region as shown in Figure 6 for six vehicles. Through settling (24) then the resulting position of these six vehicles can be reached to its final formation as shown in Figure 7. Moreover, the trajectory of these six vehicles starting from the initial positions to the final positions is shown in Figure 8, where the initial positions of the vehicles are marked with an 'o', while the final positions of the hexagon formation are marked with ' $*$ '.

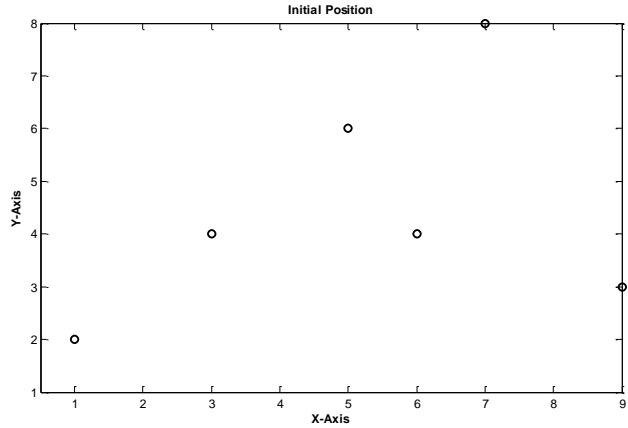

Figure 6. Six vehicles at random initial positions

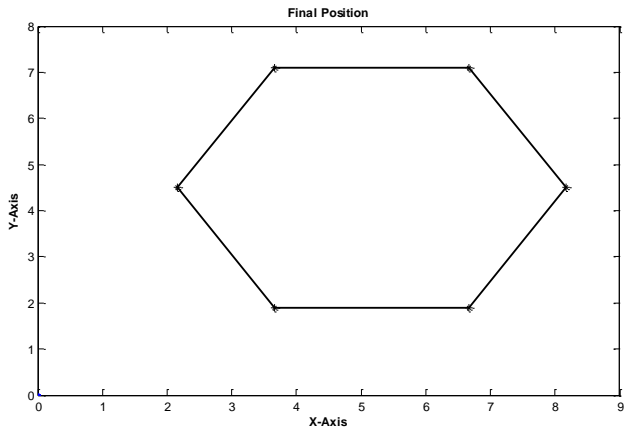

Figure 7. Six vehicles in the final formation positions

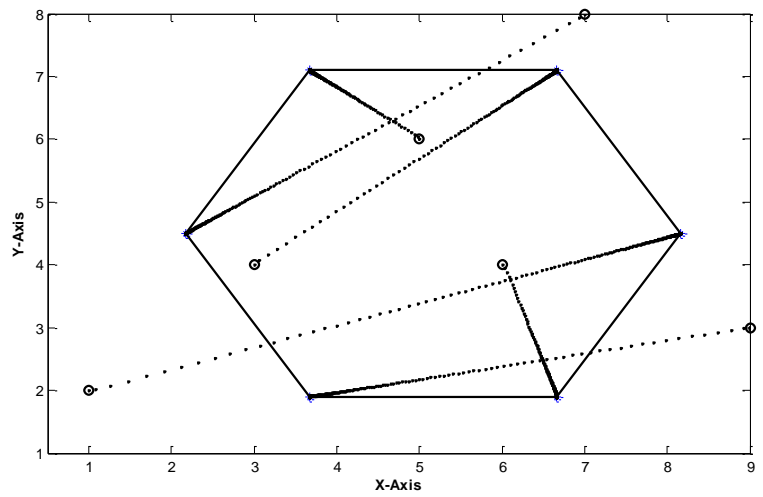

Figure 8 . Trajectory of the six vehicles in the hexagon formation

\section{OPTIMIZING THE FORMATION CONTROL OF MAS}

Graph Theory (GT) and iterative Consensus Algorithm (CA) are conducted in order to reach the two formations mentioned in previous section. At least 500 iterations are required by the vehicles in order to converge to the final position of circular and hexagon formations. Selecting suitable values for $f_{1}$ and $f_{2}$ has a vital role in attaining the required stability and accelerating the vehicles converge to the formation state. The behavior of vehicles for both the circle and hexagon formation from initial to final position are shown in Figure 9 and Figure 10 for different iterations (0, 40,80, 120, 150, and 500), respectively. In this experiment the values for $f_{1}$ and $f_{2}$ are randomly chosen. However, the selected values should keep and maintain the conditions of stability.

In order to automate the formation control and rise up the speed of convergence of the vehicles to the required formation positions (i.e. final positions). Then, Particle Swarm Optimization (PSO) is used to select the optimum values of the feed-back gains $\left(f_{l}\right.$ and $\left.f_{2}\right)$ to satisfy the indispensable and sufficient conditions. Therefore, using PSO to select the optimum values of gains $\left(f_{1}\right.$ and $\left.f_{2}\right)$ results in successful formation at 120 and 115 iterations for the circle and hexagon formation as shown in Figure 11 and Figure 12, respectively. Thus, the vehicles converge to the required final positions in minimum number of iterations, which means less cost and elapsed time. 

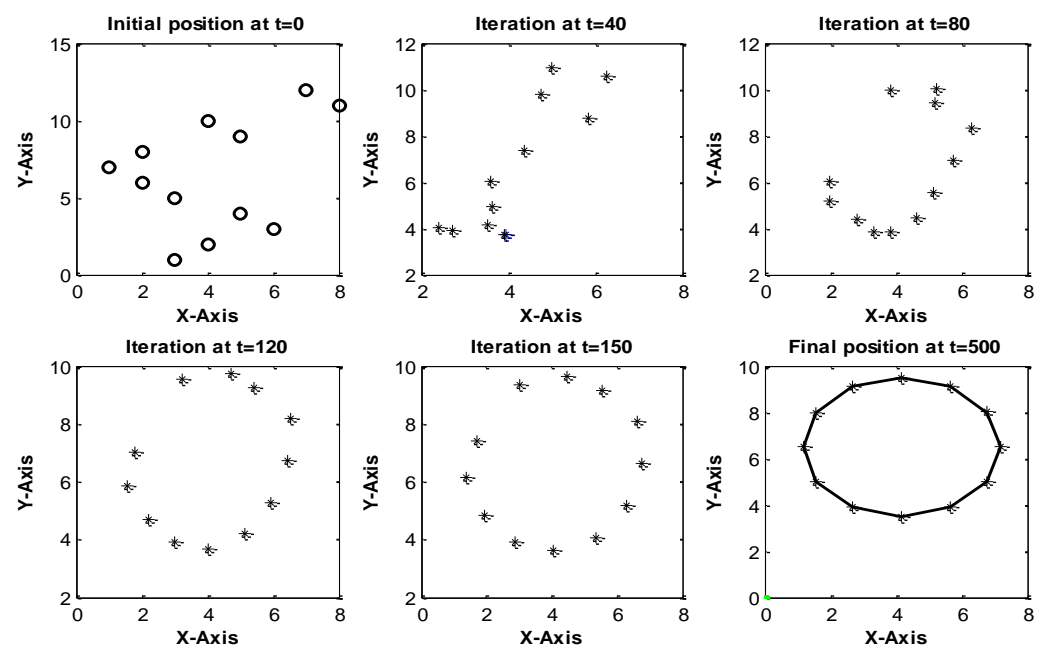

Figure 9. Vehicles formation in different iterations until final position for circle
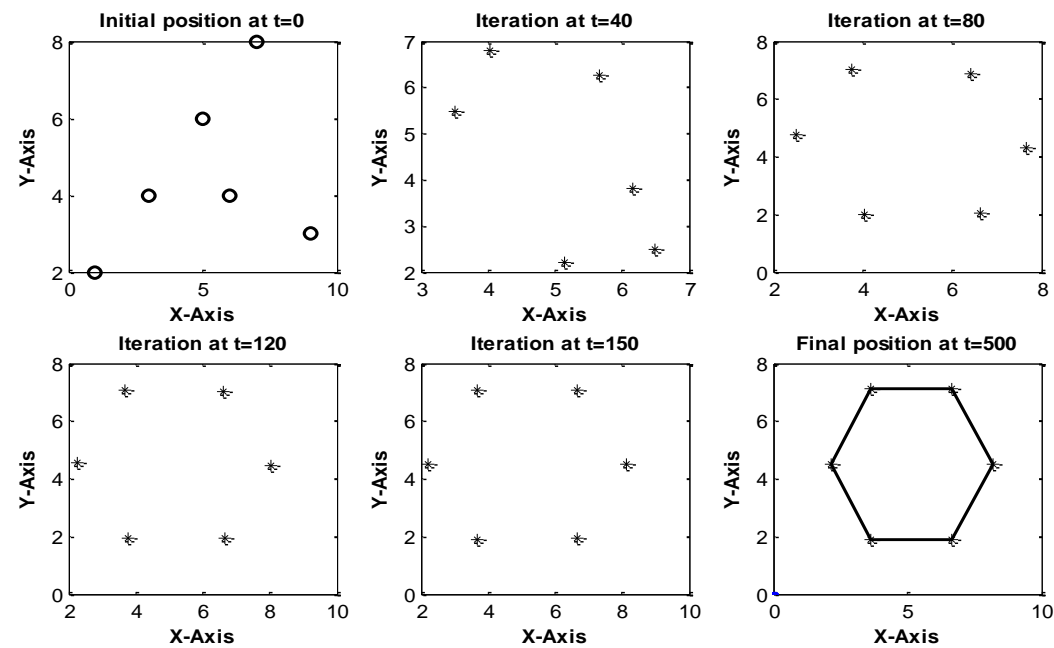

Figure 10. Vehicles formation in different iterations until final position for hexagon
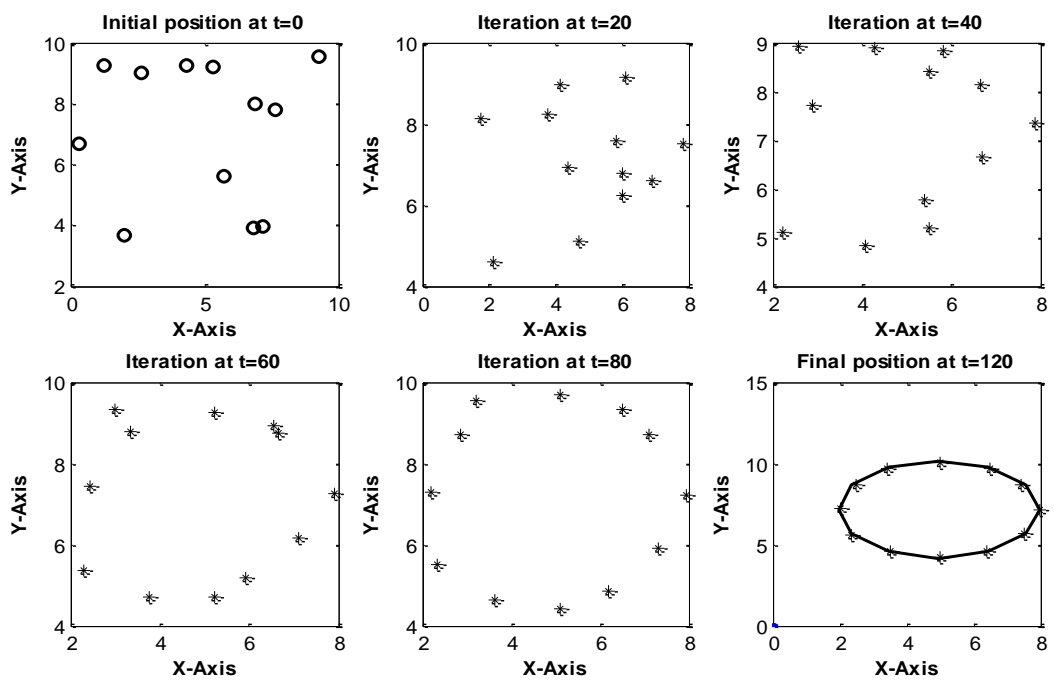

Figure 11. Optimized vehicle formation in different iterations until final position for circle 

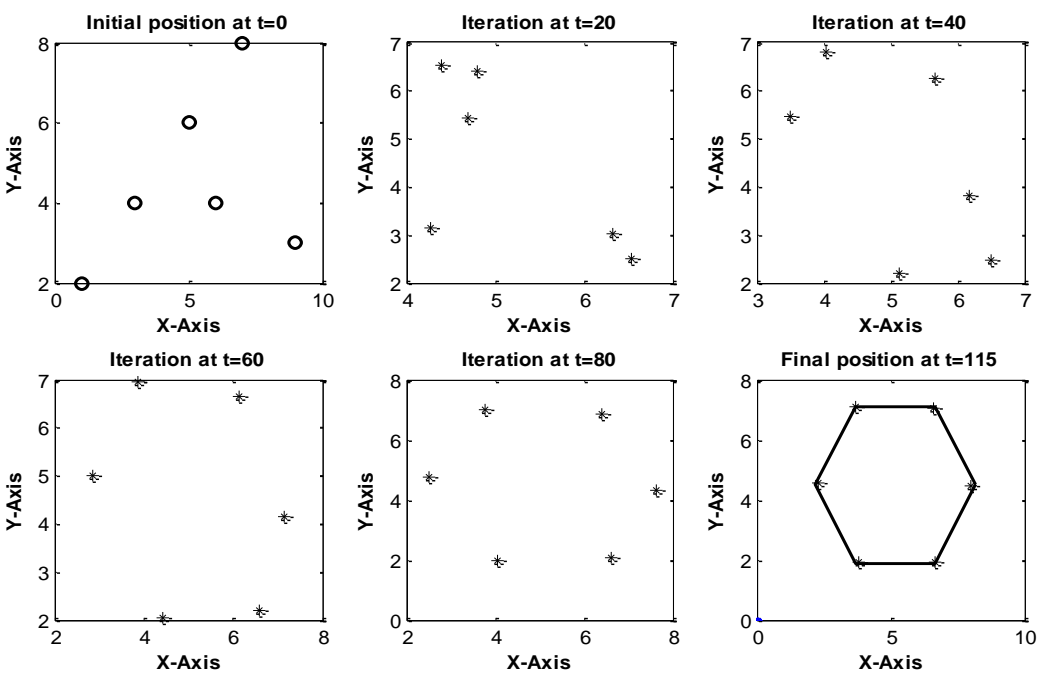

Figure 12. Optimized vehicle formation in different iterations until final position for hexagon

\section{CONCLUSION}

In this paper, the formation of multi-vehicles networks system has been developed. Different graph topologies as of a circle and hexagon have been considered. Consensus algorithm has been utilized for vector dependent. It has been noticed that formation of a particular geometry required. The main contribution of this paper is the implementation of PSO for choosing the optimal feed-back gain. Increased velocity of the vehicles in the network to shape the specified geometrical figure has been gained. The improvement of reducing the number of iterations through utilizing PSO is $76 \%$ for circle and $77 \%$ for hexagon shapes respectively. Formation tracking is considered as one of the hottest spot nowadays which can be a common problem in UAV to be focus on in the future.

\section{REFERENCES}

[1] R. O. Saber, et al., "Consensus and Cooperation in Multi-Agent Networked Systems," Proc. IEEE, vol. 95, no. 1, pp. 215-233, 2007.

[2] J. Han and Y. Chen, "Multiple UAV formations for cooperative source seeking and contour mapping of a radiative signal field," J. Intell. Robot. Syst., vol. 74, no. 1-2, pp. 323-332, 2014.

[3] J. Suh, et al., "Vision-based coordinated localization for mobile sensor networks," IEEE Trans. Autom. Sci. Eng., vol. 13, no. 2, pp. 611-620, 2016.

[4] J. A. Fax and R. M. Murray, "Information Flow and Cooperative Control of Vehicle Formations," IEEE Trans. Automat. Contr., vol. 49, no. 9, pp. 1465-1476, 2004.

[5] X. Dong, et al., "Theory and Experiment on Formation-Containment Control of Multiple Multirotor Unmanned Aerial Vehicle Systems," IEEE Transactions on Automation Science and Engineering, vol. 16, no. 1, pp. 229-238, 2019.

[6] Y. Liu, et al., "A Distributed Control Approach to Formation Balancing and Maneuvering of Multiple Multirotor UAVs," IEEE Transactions on Robotics, vol. 34, no. 4, pp. 870-881, 2018.

[7] S. K. Pang, et al., "Detection and tracking of coordinated groups," IEEE Transactions on Aerospace and Electronic Systems, vol. 47, no. 1, p. 472-502, 2011.

[8] X. Wang, et al., "Cooperative UAV formation flying with obstacle/collision avoidance," IEEE Trans. Control Syst. Technol., vol. 15, no. 4, pp. 672-679, 2007.

[9] J. Wang and M. Xin, "Integrated optimal formation control of multiple unmanned aerial vehicles," IEEE Trans. Control Syst. Technol., vol. 21, no. 5, pp. 1731-1744, 2013.

[10] Y. Cao and W. Ren, "Distributed Coordinated Tracking via a Variable Structure Approach - Part I: Consensus Tracking," 2010 American Control Conference Marriott Waterfront, Baltimore, MD, USA, 2010.

[11] J. Shin and H. J. Kim, "Nonlinear model predictive formation flight," IEEE Trans. Syst., Man, Cybern. A, Syst., Humans, vol. 39, no. 5, pp. 1116-1125, 2009.

[12] L. Y. Huang, et al., "Fast consensus algorithm of multi-agent systems with double gains regulation," Int. J. Control, vol. 7179, pp. 1-9, 2016.

[13] C. X. Yang, et al., "Fast consensus tracking of multiagent systems with diverse communication delays and input delays," Math. Probl. Eng., vol. 2014, 2014.

[14] J. Gao, et al., "Fast Consensus of Multi-agent Systems With Two-Hop Networks," MPE, Hindawi Publ. Corp., vol. 2015, 2015. 
[15] Y. Cao, et al., "An overview of recent progress in the study of distributed multi-agent coordination," IEEE Trans. Ind. Informat., vol. 9, no. 1, pp. 427-438, 2013.

[16] X. Zhao, et al., "Distributed consensus of multiple Euler-Lagrange systems networked by sampled-data information with transmission delays and data packet dropouts," IEEE Trans. Autom. Sci. Eng., vol. 14, no. 3, pp. 1440-1450, 2017.

[17] J. Xi, et al., "Admissible output consensualization control for singular multi-agent systems with time delays," J. Franklin Inst., vol. 353, no. 16, pp. 4074-4090, 2016.

[18] W. Ren, "Consensus strategies for cooperative control of vehicle formations," IET Control Theory Appl., vol. 1, no. 2, pp. 505-512, 2007.

[19] E. Rojo-Rodrigues, et al., "Implementation of a Super Twisting controller for distributed formation flight of multiagent systems based on consensus algorithms," International Conference on Unmanned Aircraft Systems (ICUAS), Miami, FL, USA, pp. 1101-1107, 2017.

[20] M. Tumari, et al., "Liquid Slosh Control by Implementing Model-free PID Controller with Derivative Filter based on PSO," Indonesian Journal of Electrical Enginnering and Computer Science, vol. 18, no. 2, 2019.

[21] H. Lim, et al., "Particle Swarm Optimization Algorithms with Selective Differential Evolution for AUV Path Planning," International Journal of Robotics and Automation, vol. 9, no. 2, pp. 94-112, 2020.

[22] Y. Merzoug, et al., "Optimal Placement of Wind Turbine in a Radial Distribution Network using PSO Method," International Journal of Power Electronics and Drive System, vol. 11, no. 2, pp. 1074-1081, 2020.

[23] M. B. Egerstedt and M. Egerestedt, "Graph-Theoretic Methods for Multi-Agent Coordination," Robomat 2007, pp. 1-10, 2007.

[24] S. Bessy, "Some problems in graph theory and graphs algorithmic theory," 2012.

[25] G. Lafferriere, et al., "Decentralized control of vehicle formations," Syst. Control Lett., vol. 54, pp. 899-910, 2005.

[26] Z. Lin, et al., "Distributed formation control of multi-agent systems using complex laplacian," IEEE Trans. Automat. Contr., vol. 59, no. 7, pp. 1765-1777, 2014.

[27] J. C. Barca, et al., "Controlling formations of robots with graph theory," Adv. Intell. Syst. Comput., vol. 194 AISC, no. 2, pp. 563-574, 2013.

[28] J. Dong, et al., "Formation Control of Multirobot Based on I / O Feedback Linearization and Potential Function," Hindawi Publ. Corp. Math. Probl. Eng., vol. 2014, pp. 1-7, 2014.

[29] T. D. Roopamala, "Comments on 'Routh Stability Criterion," vol. 7, no. 2, pp. 77-78, 2010.

[30] L. Fang and P. J. Antsaklis, "Decentralized formation tracking of multi-vehicle systems with nonlinear dynamics," 14th Mediterr. Conf. Control Autom. MED’06, 2006.

\section{BIOGRAPHIES OF AUTHORS}
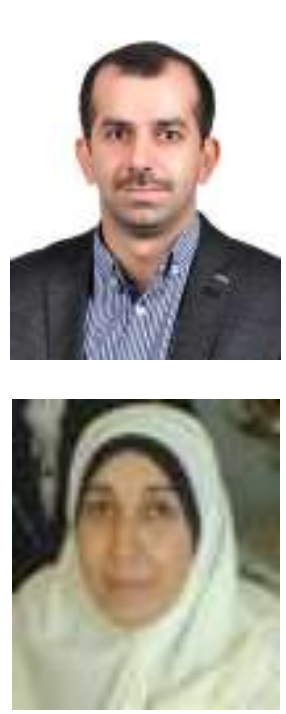

Ahmed M. Hasan received the B.S. degree in control engineering from Control and Systems engineering Dept., University of Technology (UOT) in 2002, the M.Sc. degree in Computer Control from the same university in 2006, and a Ph.D in Computer and Communication engineering in Universiti Putra Malaysia (UPM), and he is working as a research assistant in Universiti Putra Malaysia from 2008-2012, he is working as tutor and assistant lecturer in the University of Technology from 2012-to present, his research interest are Intelligent Systems, Evolutionary algorithms, Navigation Systems, Signal Processing.

Safanah M. Raafat, received her the B.Eng. and M.Sc. degrees in Control and System Engineering from the University of Technology-Baghdad, and Ph.D. from Mechatronics Engineering at the International Islamic University Malaysia. Safanah has many publications on robust control and identification of uncertainties, sliding mode control, Extremum seeking control and real-time scheduling. She is currently an Assistant Professor of the Department of Control and System Engineering at The University of Technology-Baghdad. Now she is a Senior Member of IEEE and a member of IEEE Control Systems Society. Her main research interests include System identification, optimization, and optimal control, robust control systems theory and applications, intelligent system, robotics, and positioning systems. 